\title{
Gluon polarization measurements and the possible role of diffractive process in the transverse single spin asymmetry mea- surements in RHIC-PHENIX
}

\author{
Itaru Nakagawa ${ }^{1,2, a}$ for the RHIC Spin Collaboration \\ ${ }^{1}$ RIKEN, 2-1 Hirosawa, Wako, Saitama, 351-0198, Japan \\ ${ }^{2}$ Riken-BNL Research Center, 20 Pennsylvania Avenue BIdg.510A, Upton, NY 11973, U.S.A.
}

\begin{abstract}
Two selected topics from the latest RHIC spin results are discussed here. For the transversely polarized spin program, an unexpectedly large single spin asymmetry in the very forward neutron production observed in polarized proton + nucleus collisions at $\sqrt{s}=200 \mathrm{GeV}$ is discussed in this document. For the longitudinal program, the latest highlights from the measurements on the gluon spin components of the proton spin is discussed. After a decade of continuous efforts to hunt for the gluon polarization, the RHIC collaboration is about to catch the tail of the experimental evidence that gluon carries substantially large portion of the proton spin.
\end{abstract}

\section{Introduction}

Proton is known as a strongly interacting, relativistic bound state of quarks and gluons (referred to as partons) in quantum chromodynamics (QCD). The goal of the RHIC spin collaboration is to address the open questions on the proton's spin structure which are necessary to understand the fundaments of QCD. See reference [1] for extensive discussion what have achieved and what will be explored in the future as the spin program at RHIC. The polarized proton-proton collider at RHIC provides an unique opportunity to study the spin structure of proton. The latest highlights from the RHIC spin program are selectively introduced in this document. The spin program is classified into two main programs based on the spin orientation of incident proton spin orientation with respect to its momentum direction either transverse or longitudinal. The main focus of this document discussed in section 2 is about the quite striking atomic mass dependence in the single spin asymmetry of the very forward (almost zero degree) neutron production recently observed by the PHENIX experiment. The asymmetry observed in $p+p$ was well reproduced by the interference between one pion exchange (OPE) and $a_{1}$-Reggeon. However this hadronic interference predicts only moderate dependence on the atomic mass number. I will introduce the possible mechanism which has been overlooked such as the ultra peripheral collision (UPC). For longitudinal program, the latest highlights from the measurements on the gluon spin component of the proton spin is discussed in sections 3 , respectively.

\footnotetext{
ae-mail: itaru@ riken.jp
} 


\section{The Very Forward Neutron Asymmetry}

The transverse single spin asymmetry (TSSA) of very forward (almost zero degree) was first discovered[2] at RHIC in transversely polarized proton+proton collision at $\sqrt{s}=200 \mathrm{GeV}$. Neutrons were measured by a zero-degree calorimeter (ZDC) [3] with a position-sensitive shower-maximum detector (SMD). One ZDC module is composed of $\mathrm{Cu}-\mathrm{W}$ alloy absorbers with PMMA-based communication grade optical fibers and corresponds to 1.7 nuclear interaction lengths. A single photomultiplier collects Cherenkov light via optical fibers. Three ZDC modules are located in series (5.1 nuclear interaction lengths) at $18 \mathrm{~m}$ away from the collision point, covering $10 \mathrm{~cm}$ in the transverse plane. The detector covers the psuedorapidity range of $6.8 \leq \eta \leq 8.8$ where the neutron emission angle with respect to the incident proton direction is limited to less than $2.2 \mathrm{mrad}$. Relatively large energy neutrons are selected in the analysis, i.e. the neutron energy cut was applied at the energy fraction of incident proton $x_{\mathrm{F}}=E_{\mathrm{n}} / E_{\mathrm{p}} \geq 0.4$. The production mechanism of the such a neutron is driven by soft process and of which cross section is well described by one pion exchange (OPE) model. The corresponding four momentum transfer can be defined $0.02 \leq-t \leq 0.5(\mathrm{GeV} / \mathrm{c})^{2}$ at under the assumption of OPE.

The single spin asymmetries for such a forward neutron turned out to be large[2]. Although the OPE model was quite successful to reproduce the cross section data, the predicted asymmetry within the OPE framework appeared to be very tiny and far underestimates the data[4]. The forward neutron asymmetry is formulated as

$$
A_{\mathrm{N}}=\phi_{\text {flip }} \phi_{\text {non-flip }} \sin \delta
$$

where $\phi_{\text {flip }}\left(\phi_{\text {non-flip }}\right)$ is spin flip (spin non-flip) amplitude between incident proton and out-going neutron, and $\delta$ is the relative phase between these two amplitudes. Although the OPE can contribute to both spin flip and non-flip amplitudes, resulting $A_{\mathrm{N}}$ is small due to the small relative phase. The decent amplitude can be generated only by introducing the interference between spin flip $\pi$ exchange and spin non-flip $a_{1}$-Reggeon exchange which has large phase shift in between[4].

In year 2015 , the first attempt was made to collide the transversely polarized proton and nucleus in RHIC at $\sqrt{s}=200 \mathrm{GeV}$. In order to explore the evolving origin of the asymmetry as a function of atomic mass number (A), the forward (p-going side) neutron asymmetries were measured for $\mathrm{p}+\mathrm{Au}$ and $\mathrm{p}+\mathrm{Al}$ collisions. Since the proton beam is unpolarized at LHC, the measurement is the high energy frontier of the polarized proton and nucleus collision. The existing polarized proton+nucleus experiments are carried out in one order of magnitude lower $\sqrt{s}$, for instance, at RHIC[6] and Fermi Lab.[5] using polarized proton beam and fixed nuclear targets.

The asymmetry analysis basically followed the same procedures and conditions as previous $\mathrm{p}+\mathrm{p}$ measurements. The measurement in $p+p$ collision system was repeated in year 2015 and resulting asymmetry was consistent with previous measurements. The ZDC acceptance was segmented into eight azimuthal bins and evaluated left-right raw asymmetries $\epsilon_{N}^{e x p}$ defined by the Eqn. (2) in the each bin independently.

$$
\epsilon_{N}^{r a w}(\phi)=\frac{N^{+}(\phi)-N^{-}(\phi)}{N^{+}(\phi)+N^{-}(\phi)}
$$

where $N^{+}(\phi)$ and $N^{-}(\phi)$ are number of neutrons observed in the given azimuthal $\phi$ bin when protons are polarized up and down, respectively.

Fig.1 shows preliminary results of forward neutron $A_{\mathrm{N}}$ measurements in 2015 . The red points are the results of forward neutron $A_{\mathrm{N}}$ inclusive measurements. Surprisingly, they show unexpectedly strong mass number (A) dependence. The asymmetry even flips the sign from $\mathrm{p}+\mathrm{p}, \mathrm{p}+\mathrm{Al}$ to $\mathrm{p}+\mathrm{Au}$. 
The $\mathrm{p}+\mathrm{Au}$ data point shows magnificently large $A_{\mathrm{N}}$ of about 0.18 which is three times larger than that of $\mathrm{p}+\mathrm{p}$ in absolute amplitude. The existing $\pi$ and $a_{1}$-Reggeon interference framework predicts only moderate evolution as growing $\mathrm{A}$ and does not have any mechanism to flip the sign of $A_{\mathrm{N}}$ in any $\mathrm{p}+\mathrm{A}$ collision systems.

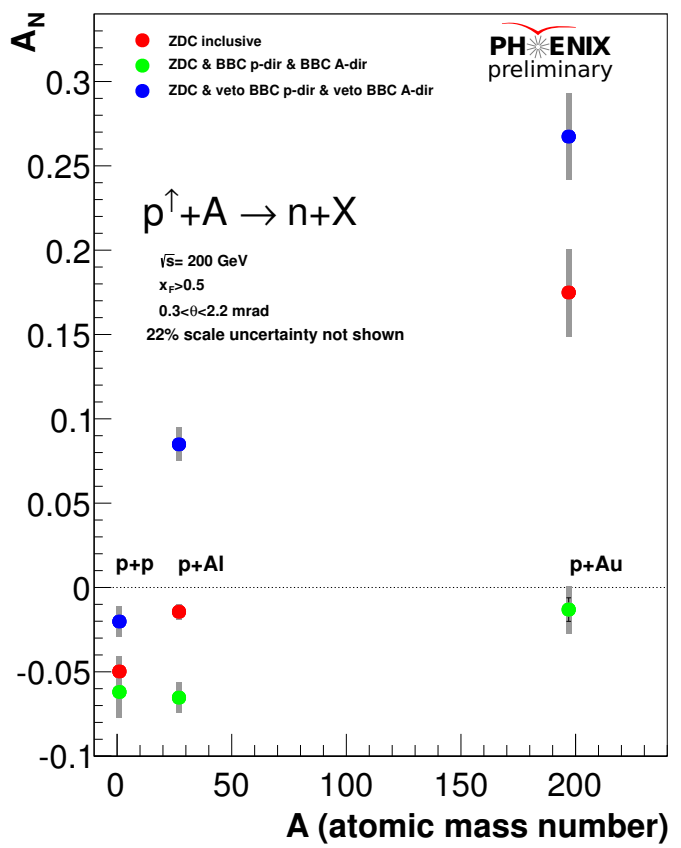

Figure 1. (Observed forward neutron $A_{\mathrm{N}}$ in transversely polarized proton-nucleus collisions. Data points are $\mathrm{A}=1, \mathrm{~A}=27$, and $\mathrm{A}=197$ are results of $\mathrm{p}+\mathrm{p}, \mathrm{p}+\mathrm{Al}$, and $\mathrm{p}+\mathrm{Au}$, respectively. Red, Blue and Green data points are neutron inclusive, neutron $+\mathrm{BBC}$ veto, and $\mathrm{BBC}$ tagged events, respectively

More interestingly, another drastic dependence of $A_{\mathrm{N}}$ was observed in correlation measurements in addition to the inclusive neutron. In these measurements, another out-going charged particle was either tagged or vetoed within the acceptance of the beam-beam counter (BBC) in both North and South arms which covers $3.1 \leq|\eta| \leq 3.9$. The BBCs cover such a limited acceptance, but the resulting asymmetries behaved remarkably contradicts. Once BBC hits (BBC tagging) are required in both arms (green data points), the drastic behavior of inclusive $A_{\mathrm{N}}$ is vanished and no flipping sign was observed between $\mathrm{p}+\mathrm{p}$ and $\mathrm{p}+\mathrm{Au}$. On the contrary, the asymmetries are pushed even more positive for $\mathrm{p}+\mathrm{Al}$ and $\mathrm{p}+\mathrm{Au}$ data points once no hits in $\mathrm{BBC}$ are required (BBC vetoed) as represented by blue data points.

It was pointed out the importance of the electro-magnetic (EM) interaction effect in the existing fixed target experiments [5] and [6], which were carried out in small $t$, one may need to consider the effect here, although it was ignored in $p+p$ data. Due to the smallness of the four momentum transfers of the present kinematics, i.e. $-t \leq 0.5(\mathrm{GeV} / \mathrm{c})^{2}$, the EM interaction may play a role which becomes increasingly important in large atomic number nucleus. The Coulomb field of the nucleus becomes rich source of exchanging photons between the polarized proton. This is known as the ultra-peripheral 
collision (UPC) in heavy ion collider experiments. In the UPC process, there is no charge exchange at the collision vertex unlike $\pi$ or $a_{1}$ meson exchange. The forward neutron in the final state then can be produced through the diagrams shown in Fig. 2.

(a)

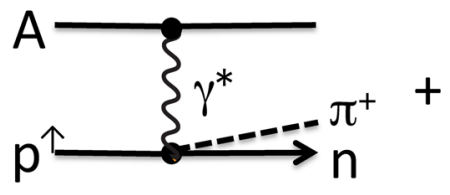

(b)

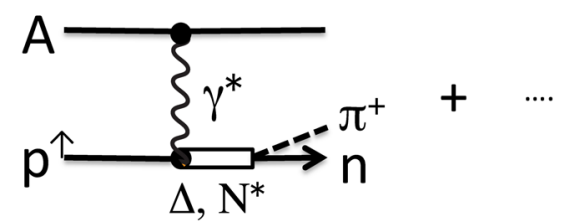

Figure 2. Feynman diagrams of UPC process to leave the high-z neutron in the final state to the proton going directions. (a) threshold photo pion production (b) $\Delta$ or $N^{*}$ excitation and its decay into $\mathrm{n}+\pi^{+}$channel.

The description of $A_{\mathrm{N}}$ is thus extended from Eqn. (1) to Eqn. 4, which includes not only hadronic but also EM amplitudes:

$$
\begin{aligned}
A_{\mathrm{N}} & =\phi_{\text {flip }}^{\text {had }} \phi_{\text {non-flip }}^{\text {had }} \sin \delta_{1}+\phi_{\text {flip }}^{\mathrm{EM}} \phi_{\text {non-flip }}^{\text {had }} \sin \delta_{2} \\
& +\phi_{\text {flip }}^{\text {had }} \phi_{\text {non-flip }}^{\mathrm{EM}} \sin \delta_{3}+\phi_{\text {flip }}^{\mathrm{EM}} \phi_{\text {non-flip }}^{\mathrm{EM}} \sin \delta_{4}
\end{aligned}
$$

where 'EM' and 'had' stand for electromagnetic and hadronic interactions, and $\delta_{1} \sim \delta_{4}$ are relative phases, respectively. The second and the third terms are known as Coulomb nuclear interference (CNI), which is observed to cause $<5 \%$ asymmetry of elastic scattering in $\mathrm{p}+\mathrm{p}$, and $\mathrm{p}+\mathrm{C}$ processes [6].

According to MC study [7], the neutron and its counter part $\pi^{+}$via UPC process is substantially boosted towards the proton beam direction and therefore the fragmenting $\pi^{+}$are mostly emitted in even higher rapidity region than BBC. Only small fraction of $\pi^{+}$are detected by BBC. Thus EM processes are suppressed in the BBC tagging events while enhanced in the BBC vetoed events. As a consequence, one may draw a hypothesis that the moderate $A_{\mathrm{N}}$ evolution of the $\mathrm{BBC}$ tagging data are consistent with the prediction based on $\pi$ and $a_{1}$-Reggeon interference scenario because the data are dominated by the hadronic amplitude. Although it is not trivial to predicts the sign of the $A_{\mathrm{N}}$ caused by EM amplitude, the observed growing feature of $A_{\mathrm{N}}$ for BBC vetoed events makes sense if EM amplitude guides to opposite sign of hadronic interactions at least qualitatively. Nevertheless it is not known at all at this moment why EM should have opposite sign nor should have such a large amplitude or large relative phases between hadronic/EM spin-flip and spin-nonflip amplitudes to produce such the large asymmetries. It remains mystery.

\section{Gluon Polarization}

Gluon polarization within the proton is probed through the measurements of double helicity asymmetry, $A_{\mathrm{LL}}$ in various production channels involving gluon dominated hard scattering processes. $A_{\mathrm{LL}}$ is defined as the difference between cross sections for colliding bunches with the same helicity and opposite helicity, divided by the sum of them. The most statistically sufficient channels are inclusive $\pi^{0}$ and jet production. Shown in Fig. 3 are inclusive $\pi^{0}$ and jet measurements by PHENIX and STAR experiments, respectively. The polarized gluon distribution function $\Delta g\left(x, Q^{2}\right)$ is extracted by fitting data based on a next-to-leading order (NLO) pQCD framework[12][13]. These global fitting 
models are confirmed to reproduce inclusive cross sections. As a consequence of the integration of so determined $\Delta g\left(x, Q^{2}=10 \mathrm{GeV}\right)$ within the range of $x>0.05$ is calculated to be $0.20_{-0.07}^{+0.05}$ at 90 $\%$ C.L. It implies the gluon carries significant fraction of proton spin 1/2 (40\% in the central value). The experimental efforts to extend the measured region to lower $x$ region where the majority of gluon exists is now underway.
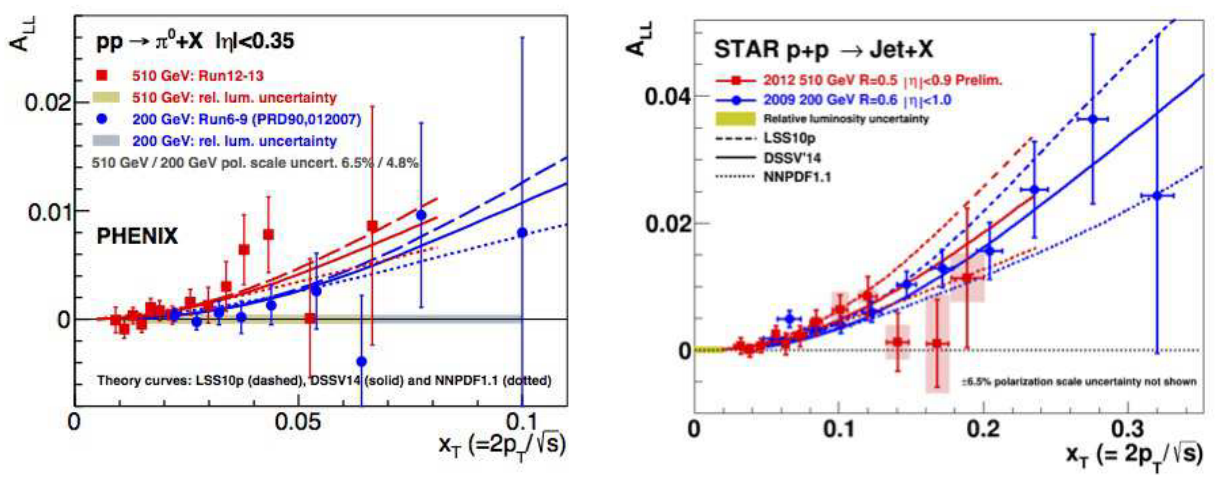

Figure 3. (left) $A_{\mathrm{LL}}$ vs. $x_{T}$ for $\pi^{0}$ production at mid-rapidity with the point-to-point uncertainties in $200 \mathrm{GeV}$ (blue circles)[8] and $510 \mathrm{GeV}$ (red squares)[9] p+p collisions, compared to NLO predictions for three recent NLO global analyses [12-14] (blue curves for $200 \mathrm{GeV}$ and red curves for $510 \mathrm{GeV}$ ). The gray/gold bands give the correlated systematic uncertainties. for inclusive $\pi^{0}$ measured in PHENIX at $\sqrt{s}=510 \mathrm{GeV}$. Theory curve is DSSV. (right) $A_{\mathrm{LL}}$ vs. xT for inclusive jet production at mid-rapidity in $200 \mathrm{GeV}$ (blue circles) [6] and $510 \mathrm{GeV}$ (red squares)[11] p+p collisions, compared to NLO predictions for three recent NLO global analyses [12-14] (blue curves for $200 \mathrm{GeV}$ and red curves for $510 \mathrm{GeV}$ ).

\section{References}

[1] RHIC The Cold Nuclear Plan for 2017 to 2023, nucl-ex, arXiv:1602.03922 and the references therein.

[2] Y. Fukao et al., Phys. Lett. B 650, 325 (2007), A. Adare et al.: Phys. Rev. D 88, 032006 (2013).

[3] C. Adler, A. Denisov, E. Garcia, M. J. Murray, H. Strobele, and S. N. White, Nucl. Instrum. Methods Phys. Res., A 470, 488 (2001).

[4] B. Z. Kopeliovich, I. K. Potashnikova, and Ivan Schmidt: Phys. Rev. Lett. 64, 357 (1990).

[5] D. C. Carey et al.: Phys. Rev. D 79, 094014 (2009).

[6] I. G. Alekseev et al.: Phys. Rev. D 79, 094014 (2009).

[7] G. Mitsuka: Eur. Phys. J. C 75:614 (2015).

[8] PHENIX Collaboration, Phys.Rev. D90 (2014) 072008.

[9] A. Adare et al. (PHENIX Collaboration), Phys. Rev. D 93, 011501R (2016) .

[10] STAR Collaboration, Phys. Rev. L115, 092002 (2015).

[11] Z. Chang, Proceeding Spin-2014, arXiv:1512.05400.

[12] D. de Florian, R. Sassot, M. Stratmann, and W. Vogelsang, Phys. Rev. Lett. 113, 012001 (2014).

[13] The NNPDF Collaboration: E. R. Nocera, R. D. Ball, St. Forte, G. Ridolfi, and J. Rojo, Nucl.

Phys. B 887 (2014) 276.

[14] E. Leader, A.V. Sidorov, and D.B. Stamenov, Phys. Rev. D 82 (2010) 114018. 